\title{
Cutaneous Vasculitis
}

National Cancer Institute

\section{Source}

National Cancer Institute. Cutaneous Vasculitis. NCI Thesaurus. Code C112210.

Inflammation of the blood vessel wall characterized by palpable purpura. 